\title{
Shoulder injuries in competitive swimmers in KwaZulu- Natal
}

\author{
T Puckree (BSc Physio, MS (Exercise Science), MEd, PhD (Exercise Physiology) ${ }^{1}$ \\ K J Thomas (B Physio) ${ }^{2}$ \\ ${ }^{1}$ Department of Physiotherapy, University of Kwazulu-Natal, Durban, and School of Physiotherapy, Sport Science and Optometry, University \\ of KwaZulu-Natal, Westville Campus, Durban \\ ${ }^{2}$ Final-year physiotherapy student, Department of Physiotherapy, University of KwaZulu-Natal, Durban
}

\begin{abstract}
Objective. To determine the incidence of shoulder injuries in competitive swimmers in KwaZulu-Natal, a province in South Africa.

Design. A cross-sectional survey was conducted. A random sample of 96 swimmers from a pool of 300 swimmers registered with first-division clubs affiliated to the KwaZulu-Natal Aquatics Association participated in the study by informed voluntary consent. Data were gathered using a validated questionnaire.

Setting. Data were gathered at time trials, races and club meetings.

Main measures. Variables monitored included the incidence of shoulder injuries, shoulder pain and proportion of overuse injury.

Results. Seventy-one per cent of the swimmers had shoulder pain and $64 \%$ reported injury to the shoulder. Forty-six per cent of the swimmers with pain complained of anterior shoulder pain, while $65 \%$ of all injuries were due to overuse. The commonest diagnoses included tendonitis $(35 \%)$, muscle imbalance $(29 \%)$, impingement $(19 \%)$ and other (17\%). Sixty-nine per cent of the swimmers swam freestyle which was related to $70 \%$ of the injuries. Eighty-one per cent of the injured swimmers sought physiotherapy for the shoulder pain.

Conclusion. The incidence of shoulder injuries in competitive swimmers is high. This study shows the need for more research into swimming injuries, and the conditioning and rehabilitation of athletes in South Africa.
\end{abstract}

\section{CORRESPONDENCE:}

T Puckree

Department of Physiotherapy

University of KwaZulu-Natal

Private Bag X54001

Durban

4000

Tel: 031-260 7977/7817

Fax: 031-260 8106

E-mail: puckreet@ukzn.ac.za

\section{Introduction}

Swimming is a popular recreational and professional sport code both locally and internationally. The South African swimming team represents South Africa at the Olympics and other world-level competitions. To ensure that these participants and those who will replace them on the world arena can function optimally, it is essential to keep them injury-free. In order to ensure that the best conditioning programmes are developed it is important to know the incidence and other related information pertaining to injury in these athletes. The shoulder joint is the most vulnerable to injury, as has been shown in many reports. ${ }^{3,6,8-11}$ To date very little research on the epidemiology of shoulder or other injuries in South African swimmers has been published in the scientific literature.

Literature from several developed countries reveals information on the incidence, ${ }^{1,2,7,8}$ and types ${ }^{1,7,8,9}$ of shoulder injuries as well as the effects of training ${ }^{7}$ on shoulder injury sustained in this sport. Most swimming injuries are due to repetitive microtrauma and overuse, with many of these injuries actually due to faulty technique. ${ }^{10,11}$ Repeated microtrauma and overuse strain of passive and active components of the shoulder lead to diminished performance over a period of time (overuse) and can lead to acute injury, resulting in reduced ability or an inability to participate in the sport. In both cases, professional training programmes, and sport-specific conditioning are crucial in determining whether participation and performance can be optimised.

When one considers the biomechanics of the shoulder joint and the demand placed on it during each of the swimming strokes, it becomes clear that a very high level of specificity in conditioning is appropriate. The structural limitations imposed by a shallow glenoid cavity together with a large degree of motion suggest the need for balanced muscle control at all times. ${ }^{13}$

The main power of propulsion is provided by arm action during the pull phase in all strokes, with the exception of breaststroke. ${ }^{14}$ Athletes, who use the arm for propelling, strain at the extremes of joint range in their drive for maximum performance. ${ }^{13}$ Repetitive motion at the extremes of range are supported by the stabilising structures or 'restraint mechanisms' which prevent excessive translation of the humeral head on the glenoid fossa. If any one of the 
mechanisms does not function properly injury will result. The rotator cuff stabilises the head of the humerus in the centre of the glenoid cavity, while the prime movers provide the power. A well-functioning rotator cuff prevents excess movement or translation of the humeral head in anterior, posterior and superior directions. Fatigue of the supraspinatus muscle predisposes the shoulder to injury due to abnormal humeral movement. ${ }^{11}$ Resistance training to increase strength of the rotator cuff and the scapular stabilising muscles has been suggested to control the risk of injury ${ }^{4}$. The stabilising structures include passive components (ligaments, joint capsule, joint cohesion mechanism, negative intra-articular pressure) and the active component (rotator cuff). Core strengthening, especially of the lower abdominal muscles, is important in supporting the trunk during arm movements. ${ }^{9}$

A search for new scientific reports on swimming-related shoulder injuries revealed just a single publication in $2003 .{ }^{9}$ In South Africa and KwaZulu-Natal (KZN) the current status of the epidemiology of swimming injuries is not known. This deficit will impact on the performance of our swimmers in the international arena. In order to address this gap, the present study looked at the incidence and causes of injuries in clublevel competitive elite swimmers in KZN.

\section{Methods}

All competitive swimmers (those who swam a qualifying time for the 2000 provincial team and achieved provincial colours) regardless of gender or race, between the ages of 13 and 25 years registered with the $\mathrm{KZN}$ Aquatics Association made up the population. From this pool of 300 swimmers, a random sample of 96 athletes were invited to participate after being fully informed and giving voluntary consent. Information on participants' personal details, medical and sporting history, training history and epidemiology of current and previous swimming-related shoulder injuries was obtained using a validated questionnaire ${ }^{17}$ with open and closed-ended questions.

The data were analysed descriptively and statistically using chi-square tests (SPSS version 6.0 for Windows). Probability was set at $p<0.05$.

\section{Results}

The response rate was $83 \%$. Eighty-one per cent of the questionnaires were usable. Forty-seven per cent of the respondents were female. Seventy-one per cent of the respondents experienced shoulder pain, while $64 \%$ reported actual shoulder injuries which included impingement, supraspinatus and bicipital tendonitis, bursitis and muscle strain. Fifty-two per cent of the injured swimmers were male. Sixty-five per cent of the injuries were due to overuse (recurrent) as opposed to traumatic injuries (25\%), which is similar to the findings reported by McMaster et al. ${ }^{12}$

Ninety-six percent of the swimmers trained and participated in events for 11 - 12 months of the year. Significantly more swimmers between the ages of 15 and 16 years (23\%) were injured compared with $14-18 \%$ in the other age categories.
Sixty-nine percent of the swimmers reported swimming freestyle. The majority $(70 \%)$ of the swimmers attributed their injuries to freestyle, which they swam most of the time.

The majority of specialist sprinters $(70 \%)$, regardless of gender, complained of shoulder injuries compared with longdistance swimmers.

The average length of a training session for a KZN swimmer was 90 minutes. The training sessions were not individualised or specific, and were unsupervised. The majority of the swimmers trained twice daily on weekdays, with an average of 11 sessions per week. In addition the majority participated in resistance training programmes. Seventy-seven per cent of the swimmers with shoulder injuries participated in resistance training (gym, strengthening exercises). The use of paddles during resistance training did not have any significant effect on the difference between those with and without injuries.

Those swimmers who reported no injury spent more time (15 minutes or more) doing stretches and warm-ups compared with 10 minutes or less by the injured swimmers. Detailed information on the stretches done was not obtained in an effort to contain the length of the questionnaire. Stretching was 1 component of the training programme.

The commonest site of pain was the anterior shoulder (55\% females and $40 \%$ males). Anterior shoulder pain was attributed to supraspinatus and bicipital tendonitis. Tendonitis (35\%), muscle imbalance (29\%) and impingement (19\%) were significantly more common than bursitis, muscle strain and laxity, which combined were responsible for the remaining $17 \%$ of injuries.

Seventy-five per cent of the swimmers who complained of shoulder injuries had to stop swimming temporarily due to the injury. Length of time away from swimming was on average 2 - 3 weeks.

Of the 52 swimmers who reported injuries, $79 \%$ received physiotherapy, which was reportedly effective for both the management and prevention of overuse injuries. No details of the specific physiotherapy modalities used were elicited. One respondent required surgical intervention for a glenohumeral joint problem.

\section{Discussion}

Sixty-five per cent of the injuries reported by elite swimmers who participated in the study were overuse (recurrent) in nature, which is similar to the findings of McMaster et al. ${ }^{12}$ This can be attributed to the fact that $96 \%$ of the swimmers trained and participated in events for $11-12$ months of the year. Therefore little time was allowed for rest or recuperation and for repair following injury. In addition, in order to be able to compete at provincial and national levels, the training schedules are extremely demanding due to the high volume, intensity, duration and frequency of training sessions. Swimming injuries involve upper-limb overuse due to the magnitude of repetitive activities. ${ }^{5,15}$ McMaster et al. ${ }^{12}$ estimate that if a swimmer swims an average of $10 \mathrm{~km}$ per day for 5 days a week and 10 months of the year, with an 
average of 15 strokes per length of a $25 \mathrm{~m}$ pool, this will translate into 1200000 repetitive arm movements per year. The KZN swimmer undertakes more than this, as shown in the results.

The fact that more swimmers between the ages of 15 and 16 years (23\%) compared with the other age categories, were injured may suggest that during this period of rapid growth, adolescents are particularly vulnerable to injuries. This is partially due to an imbalance between strength and flexibility. ${ }^{2}$ The imbalance could be in the strength between the agonists and antagonists, the relationship between flexibility and strength of the agonists and antagonists or between strength of the core stabilisers, scapular stabilisers and rotator cuff muscles which all help to stabilise during rapid mobility of the glenohumeral joint. ${ }^{9}$

The majority of specialist sprinters $(70 \%)$ regardless of gender complained of shoulder injuries compared with long-distance swimmers. This also supports the findings of Richardson. ${ }^{15}$ During a sprint, the arms turn over at a very rapid rate compared with the slower more relaxed technique employed by distance swimmers. The increased rate and speed at which the arms move overhead as well as the added power in the arm action to propel the sprinter through the water could cause repeated microtrauma to structures in the shoulder joint.

Freestyle is a very popular stroke amongst KZN competitive swimmers. The freestyle stroke utilises repetitive reciprocal overhead action. The majority $(70 \%)$ of the swimmers attributed their injuries to freestyle, which they swam most of the time. This finding is supported by the study of Richardson et al. ${ }^{16}$ which reported a $75 \%$ incidence of shoulder injuries in freestyle swimmers. The repetitive action predisposes these swimmers to injury. The fact that the swimmers' training requires more than 1 million overhead strokes per year increases their risk of shoulder injury.

The use of stretching and warm-ups prior to training seems to be effective in controlling injury rate. Stretching and warmups help to increase the range of motion, minimise incidence of musculotendinous injuries, minimise muscle soreness and reduce the risk of injury. ${ }^{7}$ Swimming is performed in a nonweight-bearing medium. ${ }^{1}$ Resistance training can increase muscular strength and reduce the incidence of overuse injuries. ${ }^{4}$ The injures sustained by $77 \%$ of the swimmers who participated in resistance training in this study could be due to problems with the manner in which the resistance exercises were performed. Therefore professional training programmes and appropriate swimming-specific supervision may help. Resistance training and conditioning or rehabilitation must be sport-specific.

Length of time away from swimming due to injury was 2 - 3 weeks, which is in keeping with suggestions by BaxterJones et al. ${ }^{2}$ who reported a recovery time of 13 days or more after an injury was sustained.

Preventive physiotherapy or teamwork between coaches, fitness specialists, physiotherapists and physicians could help swimmers overcome the burden of overuse injuries. Preventive physiotherapy should include all of the following: education on the condition; risks of the sport; motor control (in lay terms) of the shoulder action in swimming; recognition of danger signals; use of equipment; rest; balanced flexibility of the rotator cuff, scapular stabilisers, glenohumeral mobilisers and core stabilisers; strength training on land and water for the above muscle groups; core stability and endurance; cross-training for endurance and speed; and skill development through swimming-specific movement patterns. All of these will assist in adapting the muscular and neural mechanisms to optimise motor control during the sport.

\section{Conclusion}

The results of this study show that the incidence of shoulder injuries in KZN elite swimmers is significantly high. Similar to the findings of other investigators, overuse injuries predominated. This study shows the need for more research, specificity of biokinetic training and advanced levels of training and coaching to prevent or control injury rates in competitive swimmers, at least in KZN. The training programmes should include structured rest periods, cross-training that focuses on strength, endurance, speed, flexibility and co-ordination in sport-specific patterns of movement, periodisation and skill development.

\section{REFERENCES}

1. Allegrucci M, Whitney SL, Irrgang JJ. Clinical implications of secondary impingement of the shoulder in freestyle swimmers. J Orthop Sports Phys Ther 1994; 20: 307-18

2. Baxter-Jones A, Maffulli N, Helms P. Low injury rates in elite athletes. Arch Dis Child 1993; 68: 130-2.

3. Birrer P. The shoulder, EMG and the swimming stroke. J Swim Res 1986; 2(5): 20-3.

4. Fleck SJ, Falkel JE. Value of resistance training for the reduction of sports injuries. Sports Med 1986; 3(1): 61-8.

5. Fowler P. Swimmers problems. Am J Sports Med 1970; 2:141-4.

6. Fowler PJ, Webster MS. Shoulder pain in highly competitive swimmers. Orthop Trans 1983; 7(1): 170.

7. Griep JF. Swimmers shoulder. The influence of flexibility and weight training. Orthop Trans 1986; 10: 216.

8. Jones JH, Swimming overuse injuries. Phys Med Rehabil Clin N Am 1999; 10(1): 77-94

9. Johnson JN, Gauvin J, Fredericson M. Swimming biomechanics and injury prevention. Physportsmed 2003; 31(1): 1-7.

10. Kammer CS, Young CC, Niefeld MW. Swimming injuries and illnesses. Physportsmed 1999; 27(4): 51-60.

11. McMaster WC. Swimming injuries. An overview. Sports Med 1996; 22:332-6.

12. McMaster W, Troup JP, Arredondo S. The incidence of shoulder problems in developing elite swimmers. J Swim Res 1989; 5(2): 11-6.

13. Perry J. Anatomy and biomechanics of the shoulder in throwing, swimming, gymnastics and tennis. Clin J Sport Med 1983, 2: 247-70.

14. Pettrone F. Athletic Injuries of the Shoulder. New York: McGraw Hill, 1995.

15. Richardson AR. The biomechanics of swimming: the knee and shoulder Clin J Sport Med 1986; 5:103-13.

16. Richardson AR, Jobe FW, Collins HR. The shoulder in competitive swimming. Am J Sports Med 1980; 8:159-63.

17. Thomas JT. The incidence of shoulder injuries in competitive swimmers in KwaZulu Natal. Honours thesis (Physiotherapy), University of DurbanWestville, 2000, appendix 7:38-41. 